\section{The functional role of resignation orientation on goal engagement, self-esteem, life satisfaction, and depression}

\author{
Kenichi Asano, 1 Ikuo Ishimura,2 \\ Masahiro Kodama 3 \\ 1Chiba University; ${ }^{2}$ Tokyo Seitoku \\ University; 3Saitama Gakuen University, \\ Japan
}

\begin{abstract}
Several studies in Europe and North America have highlighted the importance of resignation or giving up. Research has shown that resignation is as important as goal attainment. Hence, this study examines, using path analysis, the effect of resignation orientation on goal disengagement and reengagement. Furthermore, this study attempts to clarify the implication of resignation orientation for elements of mental well-being. Questionnaires were completed by 261 Japanese college students. Results showed that proactive resignation orientation promotes reestablishment of alternative goals while negative resignation orientation encourages disengagement of the unattainable goals. The findings help explain the functional role of resignation orientation and can inform the development of treatment for resignation-related depression.
\end{abstract}

\section{Introduction}

The pursuit of goals, regarded as a form of virtue, has been related to pleasure, endurance and success. While many studies have linked successful attainment of goals with self-efficacy or optimism a myriad of socio structural, biological and normative problems make resignation from goals unavoidable. ${ }^{1}$ When confronted with unattainable goals, an individual tends to experience failure or goal stagnation, which results in reduced well-being and increased psychological distress. ${ }^{2}$ As a result, it is important to have the ability to resign from or give up the unattainable goal. In fact, most people report that goal attainment is unnecessary for satisfaction in life and that they can adapt to non-attainment of a particular goal. 3,4

Several European and North American studies have explored the importance of resignation or giving up. ${ }^{5-7}$ In Japan, resignation is primarily discussed as a coping strategy. ${ }^{8}$ The literature suggests that resignation is related to depression and other diseases, ${ }^{5,6}$ however, as mentioned in the previous paragraph, resignation can be functional and positive, as well. Asano and Kodama noted that resignation has a negative influence on stress but a positive influence on disengagement of the unattainable goal. ${ }^{9}$ This apparent discrepancy is due to differences in an individual's intention in the process of resignation. People who resign from unattainable goals bear both proactive and negative intentions, which are both essential components of resignation. The intention one has when resigning from a goal is defined as resignation orientation. It has two dimensions: proactive resignation orientation (PRO) and negative resignation orientation (NRO). ${ }^{9}$ While PRO has been associated with fewer depressive symptoms and better well-being, NRO has been associated with an increase in depressive symptoms and poorer well-being. ${ }^{9}$ PRO includes factors related to recovery while NRO reflects factors related to hopelessness and giving up. Since PRO and NRO reflect both ends of the resignation spectrum, resignation orientation potentially can explain the discrepancies in previous research regarding goal engagement and resignation.

Goal engagement consists of goal disengagement and goal reengagement, where each factor includes a set of behaviors associated with old and new goals. ${ }^{10} \mathrm{Goal}$ disengagement includes two separate factors: the disappearance of achievement desires and cognitive avoidance. Both factors are adversely related to hope. Goal reengagement includes three separate factors: setting of new goals, commitment generation, and acting on new goals. These three factors are related to hope.

Goal disengagement has been defined as the ability to withdraw efforts and commitment from an unattainable goal. ${ }^{5}$ It has been related to lower perceived stress and better self-mastery. Moreover, goal disengagement and reengagement are related to lower depression levels in parents of children actively undergoing cancer treatment. Further, goal disengagement predicts physical health. ${ }^{3}$ It has an adaptive function in older adults who faces functional disability as it reduces depressive symptoms. ${ }^{7}$ High levels of disengagement and low levels of on reengagement have been related to an increase in were found to be low on both disengagement and reengagement. 11 Therefore, the literature indicates that giving up or resignation is as important as goal attainment.

Hence, it would be of use to fill in some of the gaps missing from previous studies, which include the effect of PRO and NRO on behaviors and the relative benefit of PRO and NRO.

The present study examines the effects of resignation orientation on goal disengagement and reengagement strategies and attempt to determine how resignation affects one's self-esteem, satisfaction with life, and level of depression. Furthermore, considering
Correspondence: Kenichi Asano, Research Center for Child Mental Development, Graduate School of Medicine, Chiba University, 1-8-1, Inohana, Chuo-ku, Chiba, 260-8675, Japan.

Tel.: +81.432.262.975 - Fax: +81.432.268.588.

E-mail: kenichi.asano1225@gmail.com

Key words: resignation, well-being, students, goal disengagement.

Contributions: KA designed the study questions, conducted the statistical analyses of the data, and drafted the article; II designed the study questions and revised the article for important intellectual content; MK designed the study questions.

Conflicts of interest: the authors declare no potential conflicts of interest.

Received for publication: 15 August 2014. Revision received: 27 October 2014.

Accepted for publication: 2 November 2014

This work is licensed under a Creative Commons Attribution NonCommercial 3.0 License (CC BYNC 3.0).

CCopyright K. Asano et al., 2014

Licensee PAGEPress, Italy

Health Psychology Research 2014; 2:1882

doi:10.4081/hpr.2014.1882

gaps in the literature, this study will examine the effects and benefits of PRO and NRO on goal-related behaviors.

\section{Materials and Methods}

\section{Procedure and participants}

A total of 261 Japanese college students were recruited from three introductory psychology classes at a mid-sized university in Japan from September to November 2007. Twenty-eight questionnaires were excluded due to incomplete responses. The final sample consisted of 159 women and 74 men (mean age $=20.54$ years, $\mathrm{SD}=2.00$, range: $18-39$ years).

\section{Measures}

The main variables included measures of participants' resignation orientation, goal disengagement and reengagement, life satisfaction, self-esteem, and depressive symptoms. In addition, demographic variables (e.g., age and sex) were recorded.

Resignation orientation was measured with the Japanese Resignation Orientation Scale Revised (JROS-R), which has been validated for use with Japanese university students in previous research.12 Participants were asked to rate how frequently they had experienced 
each of the 10 thoughts related to resignation orientation on a 7 -point Likert scale $(1=$ not at all; 7 = always). The scale consists of PRO (four items) and NRO (six items) dimensions, where each dimension is computed by adding all responses of its items (Table 1). An example of the PRO dimension is I think that it is better resigning and moving on than continuing to ruminate. An example of the NRO dimension is It is restricted that I can do.

Goal disengagement and reengagement were measured by the Japanese Goal Disengagement and Reengagement Scale (JGDRS).10 This is a 14-item, self-reported questionnaire that has been validated in the literature. Participants were asked to report how they would react if they have to stop pursuing an important goal in their life. The JGDRS includes two subscales: the 11-item goal disengagement scale (e.g., It's easy for me to stop thinking about the goal and let it go) and 14-item goal reengagement scale (e.g., I start working on other new goals). The goal disengagement subscale consists of two factors: the disappearance of achievement desires and the cognitive avoidance factors. The goal reengagement subscale consists of three independent factors: setting new goals, commitment generation and acting on new goals. Each factor was computed by adding all the responses for each scale and its corresponding items (Table 1).

Life satisfaction was measured with the Satisfaction With Life Scale (SWLS) in Japanese. ${ }^{13}$ Participants were asked to rate how much they agree with each of the five items on a 7-point Likert scale ( 1 = strongly disagree 7 = strongly agree). An example of the items is In most ways my life is close to my ideal. A score consisting of these five items was computed (Table 1).

Self-esteem was measured with the Rosenberg Self-Esteem Scale in Japanese. ${ }^{14}$ Participants were asked to rate how much they agree with each of the 10 items on a 4-point Likert scale ( 1 = strongly disagree; 7 = strongly agree). An example of the items is On the whole, I am satisfied with myself. A score con- sisting of these five items was computed (Table 1).

Depressive symptoms were measured with the Center for Epidemiological Studies Depression Scale (CES-D) in Japanese. 15 Participants were asked to rate how frequently they had experienced each of 20 depressive symptoms during the past week on a 4-point scales similar to the Likert scale $(1=$ less than one day; $4=5$-7days $)$. An example of the items is I felt depressed or I felt that everything I did was an effort. A score consisting of responses for the 10 depressive symptoms was computed (Table 1).

\section{Analysis}

The calculations of the sample power were performed by the Post Hoc Compute Achieved Power analysis using the G*Power 3.16 The other statistical calculations were performed with SPSS 13.0 and Amos 4.0 for Windows. A significance level of $\mathrm{P}<0.05$ was adopted.

\section{Results}

The post hoc test to calculate the sample power of valid equations was conducted by adopting an error probability of 5\% for the sample size used. The sample power (1- $\beta$ errprob) was 0.96. Internal consistencies (Cronbach alpha) of scales in present study were sufficient (Table 1).

The correlations among variables are tabulated in Table 2. PRO was significantly correlated with setting new goal $(r=0.26, \mathrm{P}<0.01)$, commitment generation $(r=0.24, \mathrm{P}<0.01)$, acting on new goals $(r=0.27, \mathrm{P}<0.01)$, life satisfaction $(r=0.27, \mathrm{P}<0.01)$ and self-esteem $(r=0.21, \mathrm{P}<0.01)$. NR0 was significantly correlated with disappearance of achievement desires $(r=0.21, \mathrm{P}<0.01)$, cognitive avoidance $(r=0.34, \mathrm{P}<0.01)$ and depressive symptoms $(r=0.25, \mathrm{P}<0.01)$.

When path analysis was performed using maximum likelihood estimation, the fit was good, $\quad \chi^{2}(25)=54.34, \quad \mathrm{P}<0.01, \quad \mathrm{GFI}=0.96$, AGFI $=0.91, \quad$ CFI $=0.96, \quad$ RMSEA $=0.07$. The parameter estimates (Figure 1) showed that PRO had a positive effect on setting new goals $(\beta=0.26, \mathrm{P}<0.01)$, commitment generation $(\beta=0.24, \mathrm{P}<0.01)$, and acting on new goals $(\beta=0.28, \mathrm{P}<0.01)$, and had a negative effect on disappearance of achievement desires $(\beta=-17$, $\mathrm{P}<0.01)$. NRO had a positive effect on cognitive avoidance $(\beta=0.21, \mathrm{P}<0.01)$, disappearance of achievement desires $(\beta=0.37, \mathrm{P}<0.01)$

Table 1. Descriptive statistics of variables.

\begin{tabular}{lccc} 
Measure $(\mathrm{N}=233)$ & $\alpha$ & Mean & Standard deviation \\
Resignation orientation & & & \\
$\quad$ Proactive resignation orientation & 0.81 & 19.47 & 4.75 \\
$\quad$ Negative resignation orientation & 0.80 & 24.48 & 6.61 \\
Goal disengagement and reengagement & & & \\
$\quad$ Disappearance of achievement desires & 0.81 & 8.43 & 3.29 \\
Cognitive avoidance & 0.84 & 22.75 & 5.99 \\
Setting new goal & 0.90 & 18.62 & 3.92 \\
Commitment generation & 0.89 & 17.11 & 4.20 \\
Acting on new goals & 0.88 & 13.91 & 3.40 \\
\hline Life satisfaction & 0.84 & 19.39 & 6.08 \\
Self-esteem & 0.82 & 30.45 & 7.53 \\
\hline CES-D & 0.74 & 38.61 & 11.26 \\
\hline
\end{tabular}

Table 2. Correlations between variables.

\begin{tabular}{|c|c|c|c|c|c|c|c|c|c|c|}
\hline & 1 & 2 & 3 & 4 & 5 & 6 & 7 & 8 & 9 & 10 \\
\hline 1) Proactive resignation orientation & - & - & - & - & $0.26^{*}$ & $0.24 *$ & $0.27 *$ & $0.27 *$ & $0.021^{*}$ & - \\
\hline 2) Negative resignation orientation & & & $0.21^{*}$ & $0.34^{*}$ & - & - & - & - & - & $0.25^{*}$ \\
\hline 3) Disappearance of achievement desires & & & & $0.45^{*}$ & - & - & - & - & - & - \\
\hline 4) Cognitive avoidance & & & & & $0.21^{*}$ & - & - & - & - & - \\
\hline 5) Setting new goal & & & & & & $0.65^{*}$ & $0.72 *$ & - & - & - \\
\hline 6) Commitment generation & & & & & & & $0.74^{*}$ & $0.27^{*}$ & - & - \\
\hline 7) Acting on new goals & & & & & & & & - & - & - \\
\hline 8) Life satisfaction & & & & & & & & & $0.61^{*}$ & $-0.52^{*}$ \\
\hline 9) Self-esteem & & & & & & & & & & $-0.61^{*}$ \\
\hline 10) Depressive symptoms & & & & & & & & & & \\
\hline
\end{tabular}




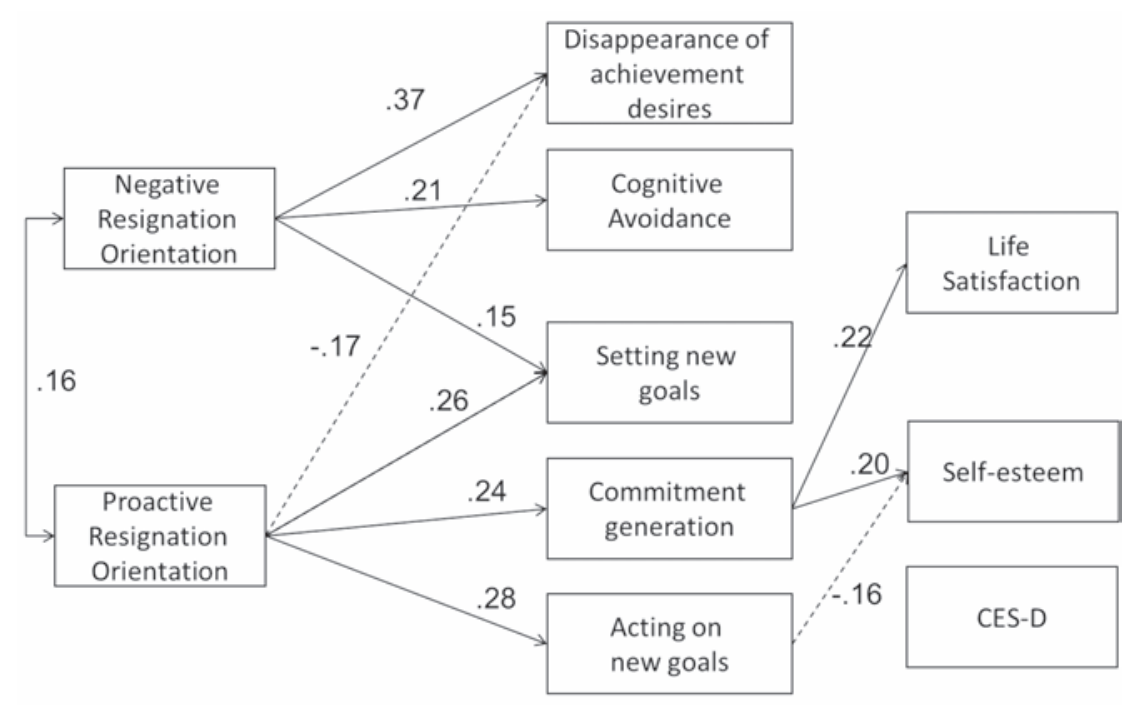

$\mathrm{GFI}=.96, \mathrm{AGFI}=.91, \mathrm{CFI}=.96, \mathrm{RMSEA}=.07 \quad \mathrm{~N}=233$

Figure 1. Path models for resignation orientation, goal disengagement and reengagement, self-esteem, life satisfaction, and depression.

and setting new goals $(\beta=0.15, \mathrm{P}<0.01)$. Commitment generation had a positive effect on life satisfaction $(\beta=0.22, \mathrm{P}<0.01)$ and selfesteem $(\beta=0.20, \mathrm{P}<0.01)$. Acting on new goals had a negative effect on self-esteem $(\beta=-0.16$, $\mathrm{P}<0.01)$.

\section{Discussion and Conclusions}

The present study showed the relationships between proactive and negative intentions and resignation, goal disengagement and reengagement, and indicators of mental well-being. Each resignation orientation exerted a unique influence on goal disengagement and reengagement.

PR0 positively affected three aspects of goal re-engagemen. This reflects the functionality of PRO and supports previous findings that PRO is associated with optimism and hope. ${ }^{9,17}$ As observed, PRO encourages reengagement of new goals. In addition, PRO negatively affects the disappearance of achievement desires (i.e., increases achievement desires). Hence, PRO may have facilitated resignation by reengaging the participants towards new goals.

NRO positively affected two aspects of goal disengagement. This result reflects the functionality of NRO and supports previous findings that $\mathrm{NRO}$ is associated with pessimism.9,17 As observed, NR0 promotes disengagement of goals. However, NRO positively affects the setting of new goals (i.e., leads to increased setting of new goals). As such, NRO may play a role in disengagement of unattainable goals and increase the likelihood that an individual may search for alternative goals.

An examination of the effect of resignation on mental well-being shows that, commitment generation and acting on new goals may affect mental well-being. Commitment generation was positively correlated with life satisfaction and self-esteem. This means that when an individual doesn't commit to a new goal without commitment, the individual will likely have lower self-esteem and life satisfaction. The results also show that acting on new goals reduces one's self-esteem. This suggests that superficially obtaining new goals can lead to lower self-esteem.

The present findings indicate that PRO and NRO have different yet complementary roles. NRO can lead to behavioral, cognitive or emotional disengagement, which is necessary for confronting unattainable goals. Subsequently, a typical progression involves recovering from the episode and searching for alternative goals. At this point, PRO comes into play. PRO involves setting of new goals, committing to the goals, and making efforts to attain the new goals. PRO was previously found to influence emotionality. 18 Considering the results of this study, PRO may promote positive emotional experience through the engagement of new goals.

Despite these contributions, some research limitations should be noted. First, the data were from self-reported questionnaires and the study's cross-sectional design does not permit causal inferences to be made. Second, generalization of the results beyond the current sample of Japanese student needs to be done with caution. Third, random sampling was not used. In addition, this study did not control for certain variables, including socioeconomic sta- tus, religion, gender roles, and experience with resignation. It would be useful to examine the influence of these variables in future studies to better understand processes associated with resignation. Consequently, the validity of the current study's results could be investigated through replication. However, the same constructs could be measured differently, for example, by interviewing people who are struggling with problems.

In this study, the relationships between resignation orientation, goal disengagement and reengagement, and mental well-being were revealed in Japanese college students by using questionnaires. Results show that PRO promotes recovery from the event and engagement towards new goals while NRO encourages disengagement of the unattainable goals. Although further research is needed to validate these findings, the current study has contributed towards understanding the concepts associated with resignation and potentially can be used for developing treatment for resignation-related depression.

\section{References}

1. Heckhausen J, Schulz R. A life-span theory of control. Psychol Rev 1995;102:284-304.

2. Carver CS, Scheier MF. Origins and functions of positive and negative affect: a control-process view. Psychol Rev 1990;97:1935 .

3. Sprangers MA, Schwartz CE. Integrating response shift into health-related quality of life research: a theoretical model. Soc Sci Med 1999;48:1507-15.

4. Brandtstädter J, Renner G. Tenacious goal pursuit and flexible goal adjustment: explication and age-related analysis of assimilative and accommodative strategies of coping. Psychol Aging 1990;5:58-67.

5. Wrosch C, Scheier MF, Miller GE, et al. Adaptive self-regulation of unattainable goals: goal disengagement, goal reengagement, and subjective well-being. Pers Soc Psychol Bull 2003;29:1494-508.

6. Wrosch C, Miller GE, Scheier MF, De Pontet SB. Giving up on unattainable goals: benefits for health? Pers Soc Psychol Bull 2007;33:251-65.

7. Dunne E, Wrosch C, Miller GE. Goal disengagement, functional disability, and depressive symptoms in old age. Health Psychol 2011;30:763-70.

8. Suzuki S. The validity of the three dimensional model to classify coping behavior. Shinrigaku Kenkyu 2004;74:504-11. [Article in Japanese].

9. Asano K, Kodama M. Relationships between resignation orientation, opti- 
mism and automatic thoughts. J Hum Care Stud 2009;10:77-86. [Article in Japanese].

10. Asano K, Kodama M. Development of a Japanese goal disengagement and reengagement scale. Tsukuba Psychol Res 2009;37:99-105.

11. Neter E, Litvak A, Miller A. Goal disengagement and goal re-engagement among multiple sclerosis patients: relationship to well-being and illness representation. Psychol Health 2009;24:175-86.

12. Asano K, Kodama M. The effect of resignation orientation in goal setting. 10th International Congress of Behavioral
Medicine 2008;218-9.

13. Sumino Z. Development of Japanese version of the satisfaction with life scale [translated by author]. Annu Conv Japn Assoc Educ Psychol 1994;36:192. [Article in Japanese].

14. Yamamoto M, Matsui Y, Yamanari Y. The structure of perceived aspects of self. Japanes J Educ Psychol 1982;30:64-8. [Article in Japanese].

15. Shima S, Kano T, Kitamura T, Asai M. A self-report depression scale for research in the general population. Seishin Igaku 1985;27:717-23. [Article in Japanese].

16. Faul F, Erdfelder E, Lang AG, Buchner A.
$\mathrm{G}^{*}$ Power 3: a flexible statistical power analysis program for the social, behavioral, and biomedical sciences. Behav Res Methods 2007;39:175-91.

17. Asano K, Kodamam. The effect of resignation orientation in goal setting. 10th Int Congr Behav Med. Tokyo: 10th International Congress of Behavioral Medicine; 2008:218-9.

18. Asano K, Hatori K, Kashimura M, Ishimura I. The relations between resignation orientation, feeling experience style and mental health. J Hum Care Stud 2013;13: 101-10. 